\title{
Análisis matemático para el diseño de Toro de Arado Biomecánico de alta Calidad
}

\section{Mathematical analysis for the design of High Quality Biomechanical Plow Bull}

Eder Lenin Cruz Siguenza. ${ }^{1}$, Wilson Javier Villagrán Cáceres. ${ }^{2}$, Elvis Enrique Arguello. ${ }^{3}$ \& Edison Patricio Abarca Pérez. ${ }^{4}$

\begin{abstract}
.
DOI: https://doi.org/10.33262/cienciadigital.v3i2.478

This study on a biomechanical plowing bull is due to the fact that vertebrates in general, and the human species in particular, movement implies the displacement of skeletal elements that are considered as passive elements. But this movement is due to active elements which are responsible for transforming chemical energy into mechanical energy or force, these active elements are muscles.

Another reason for this study is that currently the need to technify the plows are of great importance and the machines used for these jobs have very high costs, and even more there are places where you need to perform a plow that measures restricted, for which a machine of considerable acceptable size is needed and also with acceptable costs, with the characteristic that provides the same service but at lower cost. M., \& Redín, M. I. (2008). the calculations give us very concise data that will be used for

\footnotetext{
${ }^{1}$ Escuela Superior Politécnica de Chimborazo, Facultad de Mecánica, Riobamba, Ecuador, eder.cruz@espoch.edu.ec

${ }^{2}$ Escuela Superior Politécnica de Chimborazo, Facultad de Mecánica, Riobamba, Ecuador, wvillagran@espoch.edu.ec,

${ }^{3}$ Escuela Superior Politécnica de Chimborazo, Facultad de Mecánica, Riobamba, Ecuador, e_arguello@espoch.edu.ec,

${ }^{4}$ Escuela Superior Politécnica de Chimborazo, Facultad de Mecánica, Riobamba, Ecuador, edison.abarca@espoch.edu.ec
} 
the manufacture of the biomechanical bull that should have minimum characteristics such as frontal weight Pf: $270 \mathrm{Kg}$, previous weight: $180 \mathrm{Kg}$ for which the discharge of the weight in the knees per extremity With these characteristics you will obtain a maximum force in the frontal part of $295 \mathrm{Kg}$ and the previous force will be a maximum of $250 \mathrm{Kg}$, you will have to unload a weight, and finally you have to consider the dynamic system which it is with a high speed of $0.625 \mathrm{rad} / \mathrm{sec}$, and low speed of $0.4335 \mathrm{rad} / \mathrm{sec}$, and its accelerations of $0.3112 \mathrm{rad} / \mathrm{s}^{2}$, for the high and 0.45 $\mathrm{m} / \mathrm{sec}^{2}$. for low acceleration with this data you can build a prototype with a high efficiency.

Keywords: Angular, Angular Speed, Biomechanical, Forces, Plow.

\section{Resumen.}

Este estudio sobre un Toro de arado biomecánico se debe a que los vertebrados en general, y la especie humana en particular, el movimiento implica el desplazamiento de elementos esqueléticos que son considerados como elementos pasivos. Pero este movimiento se debe a elementos activos los cuales son los encargados de transformar la energía química en energía mecánica o fuerza, estos elementos activos son los músculos.

Otro motivo de este estudio se debe a que en la actualidad las necesidades de tecnificar los arados son de gran importancia y las maquinas que se utiliza para esos trabajos tienen costos muy elevados, y más aún hay lugares donde se necesita realizar un arado que tienen medidas restringidas, por lo cual se necesita una máquina de tamaño considerablemente aceptable y también con costos aceptables, con la característica que brinde el mismo servicio, pero a menor costo. M., \& Redín, M. I. (2008). Los cálculos nos dan datos muy concisos que servirán para la fabricación del toro biomecánico que deberá tener características mínimas como Peso frontal Pf: $270 \mathrm{Kg}$, Peso anterior: $180 \mathrm{Kg}$, para lo cual la descarga del peso en las rodillas por extremidad será de $140 \mathrm{Kgf}$, y $90 \mathrm{Kgf}$ respectivamente, con estas características obtendrá una fuerza máxima en la parte frontal de $295 \mathrm{Kg}$ y la fuerza anterior ser una máxima de $250 \mathrm{Kg}$, deberá descargar un peso, y finalmente se debe considerar el sistema dinámico el cual es con una velocidad alta de $0,625 \mathrm{rad} / \mathrm{seg}$, y velocidad baja de $0,4335 \mathrm{rad} / \mathrm{seg}$, y sus aceleraciones de $0,3112 \mathrm{rad} / \mathrm{s}^{2}$, para la alta y $0,45 \mathrm{~m} / \mathrm{s}^{2}$. para la aceleración baja con estos datos se podrá construir un prototipo con una alta eficiencia

Palabras claves: Aceleración angular, Arado, Biomecánico, Fuerzas, Velocidad Angular

\section{Introducción.}

menciona que la dinámica es el estudio del movimiento de un objeto, y de las relaciones de este movimiento con conceptos físicos tales como la fuerza y la masa en otras palabras 
entendemos que la dinámica intenta establecer cuáles son las causas de los movimientos, es decir, cuáles son las relaciones entre el movimiento que se produce en un cuerpo y las fuerzas que lo producen, existen dos tipos de fuerzas: Javier Junquera (2010)

Fuerzas Internas: son aquellas que ejercen unas partes del cuerpo sobre otras. Son fuerzas internas: la fuerza de tracción muscular y las fuerzas de resistencias pasiva de órganos y tejidos.

Fuerzas Externas: son aquellas que ejercen los elementos que no forman parte del sistema locomotor como son: Fuerza de la gravedad, fuerza normal y fuerza de rozamiento. M., \& Redín, M. I. (2008).

Debido a la existencia de estas fuerzas y a la herramienta que tenemos que es la dinámica se procede al estudio de los animales cuadrúpedos los cuales poseen características específicas de cada uno, con esto se analiza animales cuadrúpedos como perros, bueyes, vacas, cerdos M., \& Redín, M. I. (2008).

Los cuales tienen pesos distintos y por ende estructuras Oseas específicas de cada especie. Como el enfoque está en los animales de tiro o de arado se llega a la idea principal de que: Los animales de tiro hacen una importante contribución a la agricultura y a las economías rurales de los países en desarrollo y seguirán haciéndolo durante muchos años más. El aporte energético animal en los países del tercer mundo es equiparable a los tractores, pero su costo es mucho menor en términos económicos y ambientales. Hetz, E., \& Pérez, R. (1994).

Los animales de tiro se vienen utilizando desde milenios, tras la revolución neolítica que permitió la ganadería, especialmente cuando, más adelante, la Edad de los Metales posibilitó la tecnología de la rueda, aunque dependiendo de los medios, las culturas y otras circunstancias, se emplean otras técnicas de tiro como el trineo. Chirgwin, J. C. (1995). Los principales animales de tiro son los équidos (caballos, burros y mulas) y los bóvidos (bueyes).

Los perros tienen una utilización mucho más versátil, incluso limitándose al contexto de su uso productivo en entornos rurales (caza, pastoreo, etc.), además de ser también animales de tiro y de compañía. Alberdi, M. T., \& Prado, J. L. (1995). Los camélidos suelen utilizarse como animales de carga tanto los del viejo mundo (camellos y dromedarios) como los del nuevo mundo (llamas, vicuñas, alpacas); mientras que los renos suelen utilizarse como tiro de trineos. Los elefantes se utilizan también para tareas pesadas (desbrozar o arrastrar troncos). Fernández-Baca,S. (1991).

Diferentes autores se han ocupado de la filosofia del trabajo, efecto del medio ambiente, potencia de tiro, selección de animales par tiro, alimentación, manejo sanitario y adiestramiento. De acuerdo con ellos, el trabajo y la temperatura ambiente afecta signos vitales de los animales como temperatura corporal, ritmo respitratorio, pulsaciones y tiene 
efecto, en condiciones extremas, en la rumia, la produccion de secrecciones acuosas, la fuerza de tiro y la velocidad, por lo cual se hace necesario intercalar periodos de reposo en medio del trabajo para mantener los anteriores parámetros en valores aceptables. Ordóñez, M. V., López, M. S, (1995). La conformacion de los animales ( tamaño, masa, proporcion entre los cuartos delanteros y traseros, longitud de la línea, profundidad, anchura, musculatura, grosor de piel, anatomía de las extremidades, calor ), es muy importante para la potencia de tiro, la cual para animales de 500 a $900 \mathrm{~kg}$ puede alcanzar valores de $0.56 \mathrm{kw}$ y un tiro de $70 \mathrm{~kg} \mathrm{a}$ una velocidad de 2.88km/h . González Valdés, R., García de la Figal Costales.(2009).

La mayoria de investigadores supone que los bueyes pueden trabajar durante 6 a 7 horas al día y 300 días al año; estiman el potencial máximo de trabajo en 2000 horas y expresan las horas de trabajo reales como porcentaje de potencial máximo, parámetro que se conoce con el nombre de eficiencia de utilización; y que en la india oscila entre el 16 y el $45 \%$. Chirgwin, J. C. (1995). Los animales empiezan a trabajar entre los tres y tres años y medio y hasta los 11-15 años, estimándose el promedio en 9 años.

Por otra parte, el costo de una yunta de bueyes es el 5\% del costo de un tractor mediano, incluyendo la herramienta, el yugo y los arneses y su valor de salvamento es de mas del $60 \%$ del costo inicial. Respecto al costo de preparacion de suelos en condiciones de ladera tiene un promedio de $20 \%$ menor e ¿con tracción animal, comparado con el costo de la labranza con tractor. El presente trabajo tiene la pretension de elaborar un diagnóstico del estado actual de desarrollo de la traccion animal. Barreiro, M. W. (2017).

\section{Marco teórico}

La biomecánica se define como la ciencia y tecnología de los movimientos simples y complejos que pueden ejecutar los animales para armonizar con sus restricciones anatómicas. Elvira, J. L. L., Garcia, F. J. V.(2008). El diseño adaptivo de un organismo que se mueve autónomamente permite la ejecución de ciertas secuencias esqueleto-musculares y prohíbe ciertos movimientos "imposibles". Los movimientos tienen una fuerte relación con outputs cerebrales, sobre todo en el caso de manos que escriben signos y dibujos, que ya escapan a los límites de la biomecánica propiamente dicha. Barreto Andrade, J., Villarroya-Aparicio, A.(2017).

Cualquier restricción corporal está delimitada

por las posibilidades biomecánicas.

Teóricamente, el $60 \%$ del peso corporal del animal cuadrúpedo corresponde a la parte anterior del animal (debido al peso de la cabeza), y el $40 \%$ a la posterior. Machuca Mendoza, C. R., \& López Ayala, L. F. (2015). 
Figura1: Distribución de peso de un buey

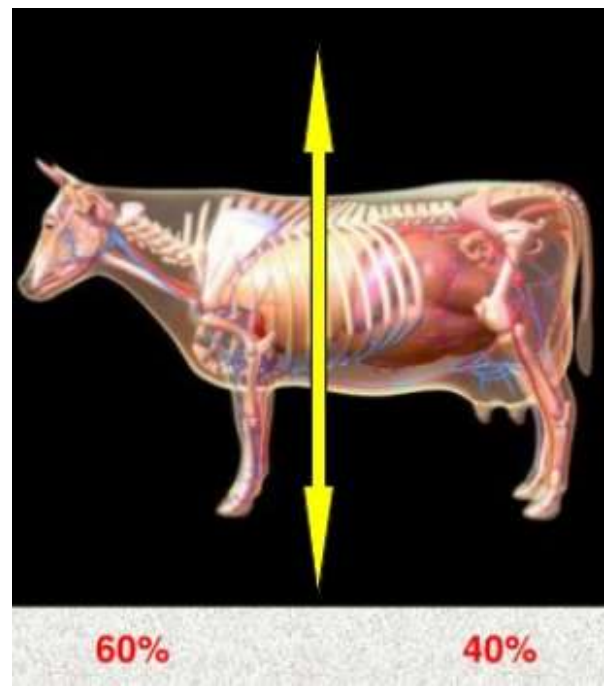

Fuente: Mejias J. (2014).

\section{Metodologia.}

La siguiente investigación es de carácter "Deductivo" ya que respondiendo a la estructura de esta clase de metodología de investigación, partimos de un conocimiento general sobre los diferentes campos en los que se encuentra inmerso nuestro trabajo investigativo y practico, como lo es el diseño de máquinas, cálculo de trabajos potencia y energías, para posteriormente tras la recopilación de información, síntesis de la misma y los diferentes experimentos y análisis de los resultados de los realizados con la maquina ya concluida, nos dan un saber más particular y especifico acerca de las propiedades y características que ha de tener nuestra máquina para una mejor eficiencia y rentabilidad.

\section{Localización Geográfica}

De acuerdo con la ubicación geográfica de los terrenos donde se busca aplicar este Toro de Arado, se debe considerar pues; la altura del terreno sobre el nivel del mar, el ángulo de inclinación del terreno y el tipo de tierra que existe en el terreno porque según eso se debe analizar la resistencia que cada tipo de tierra va a presentar, a más de eso se debe considerar la humedad del ambiente porque eso va a afectar a los mecanismo del toro de arado.

Debemos analizar con que frecuencias se presentar lluvias debido a que una tierra con mayor humedad presenta una mayor resistencia y por ende el toro debe realizar más esfuerzo para cumplir su objetivo. Mehuys, G. R., Tiessen, K. H., Villatoro, M., Sancho, F.(2009). 
Figura 2: Toros arando en una montaña

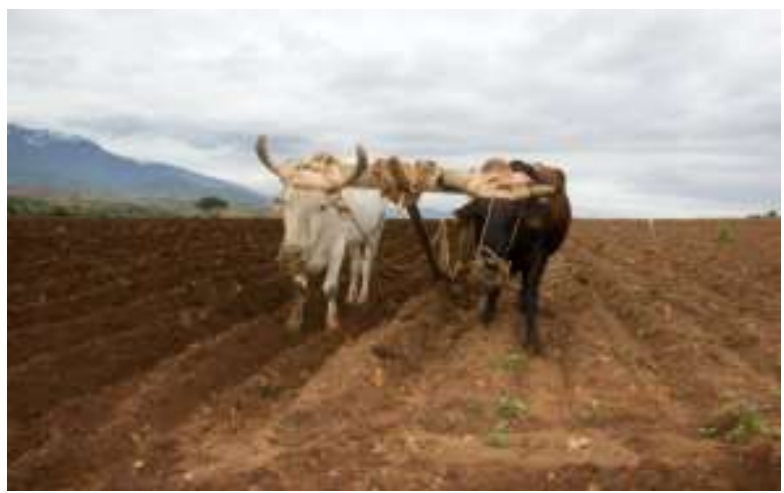

Fuente: Gobierno de México (2019)

\section{Metodología de Análisis}

Con el objetivo de cumplir cada uno de los objetivos se tomó en cuenta tres puntos de gran importancia:

1. La distribución del peso de un buey de arado, por ejemplo, un animal de $600 \mathrm{~kg}$, va a tener $360 \mathrm{~kg}$ en su parte frontal y $240 \mathrm{~kg}$ en su parte posterior.

2. La fuerza que va a soportar cada extremidad de toro de arado, debido a que esa va a ser la fuerza mínima que tendrá que soportar cada extremidad

3. La resistencia que va a presentar ciertos tipos de terrenos, para según esos datos calculas la fuerza que debe generar el toro de arado para cumplir con su objetivo.

Figura 3: Estructura ósea del buey

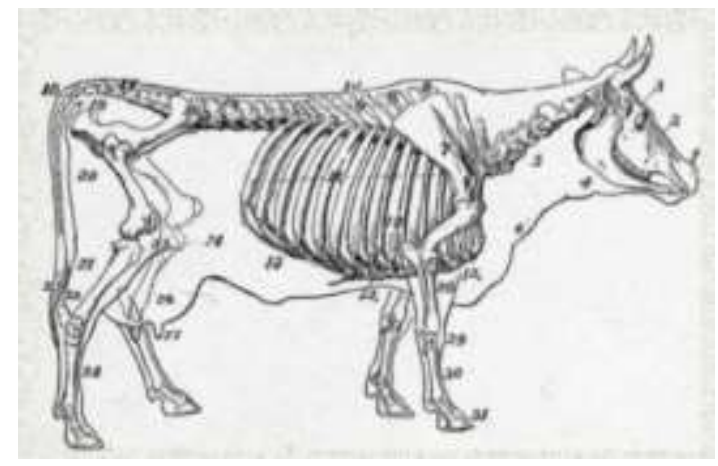

Fuente: García J (2019) 


\section{Procedimiento para el desarrollo del estudio}

- Analizar los pesos promedios de los toros de arado

- Estimar las fuerzas soportadas por cada extremidad del toro biomecánico dependiendo de los pesos calculados.

- Calcular la velocidad que se desea generar en cada toro biomecánico dependiendo de su peso.

- Analizar y seleccionar las mejores uniones para las articulaciones de las patas de la

- Analizar y seleccionar los mecanismo que proporcionen una mejor ventaja mecánica.

- Analizar y seleccionar los mejores materiales para las extremidades y las demás partes de la máquina.

- Analizar y ubicar el mejor lugar para la colocación del tren de arado.

- Proceder a la unión de cada una de las partes y mecanismos.

- Realizar pruebas de movimiento en un lugar plano para calibrar la máquina de la mejor manera.

- Proceder a las pruebas en un terreno que presente una inclinación considerable y una resistencia promedio.

Figura 4: Ejemplo de cálculo de fuerza mínima soportada por una extremidad

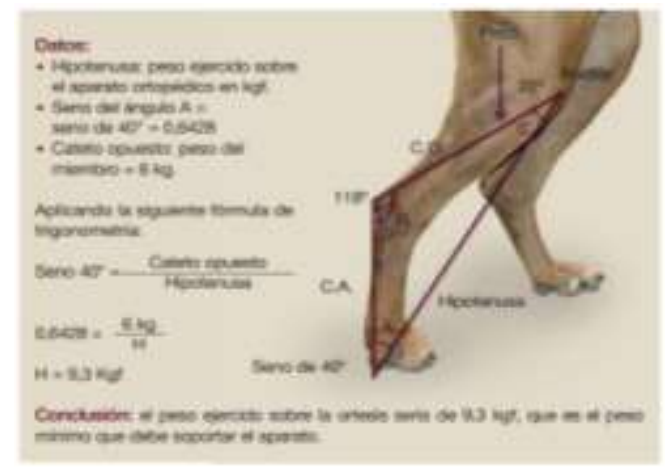

Fuente: Ivot (2019)

\section{Hipótesis:}

El planteamiento de la hipótesis de investigación e hipótesis nula se planteó de la siguiente forma:

Hi: La elaboración de un análisis matemático de calidad servirá para el diseño de un prototipo, rígido y totalmente operacional ayudará a la elaboración de un Toro de arado Biomecánico funcional y que ayudará a las labores de tiro en el arado. 
Ho: La elaboración de un análisis matemático de calidad servirá para el diseño de un prototipo, rígido y totalmente operacional ayudará a la elaboración de un Toro de arado Biomecánico funcional y que ayudará a las labores de tiro en el arado.

\section{Resultados.}

Los analisis que se enmarca la investigacion son basados en un Toro o mula de arado de constextura media capaz de realizar trabajos pesados en la agricultura.como podemos observar

Cuadro 1: Capacidad de tiro y velocidades promedio

\begin{tabular}{|c|c|c|c|c|c|c|c|}
\hline \multirow{2}{*}{$\begin{array}{l}\text { Caractarfati } \\
\text { eas del buefy }\end{array}$} & \multirow{2}{*}{$\begin{array}{c}\text { Prse adulte } \\
\text { (ke) }\end{array}$} & \multicolumn{3}{|c|}{ Velocidad baja } & \multicolumn{3}{|c|}{ Velocidad =dia } \\
\hline & & $\begin{array}{c}\text { Velecidad } \\
(\mathrm{k}=/ \mathrm{h})\end{array}$ & $\begin{array}{c}\text { Esfuerab } \\
\text { traccides } \\
\text { (kgf) } 1\end{array}$ & $\begin{array}{c}\text { Fueras } \\
(\mathrm{NW}) \text { 21 }\end{array}$ & $\begin{array}{c}\text { Velocidad } \\
(h=/ h)\end{array}$ & $\begin{array}{r}\text { Esfuerzo } \\
\text { traceibin } \\
\text { (kgf) 1 }\end{array}$ & $\begin{array}{r}\text { Fuerta } \\
(\mathrm{kW}) 2 /\end{array}$ \\
\hline Ligere & 210 & 2.3 & 30 & 0.25 & 4,0 & 21 & 0.23 \\
\hline Medio & 430 & 2,3 & 61 & 0,44 & 6,0 & 45 & 0.50 \\
\hline Fesado & 900 & 2,3 & 129 & 0,49 & 4,0 & 90 & 0,99 \\
\hline
\end{tabular}

Fuente: Rodríguez E (1986)

Tambien tomamos en cuenta el tipo de sustrato y compocición del suelo donde se puede realizar el trabajo de arado, con lo cual tomaremos datos relevantes de la preparacion y del tipo de suelo relativamente promedio que se encuentra en la zona de sud américa que nos servirá para los calculos que sustentan las cargas, como lo vemos a continuación:

Cuadro 2: Preparación de lotes de acuerdo con el estado del suelo

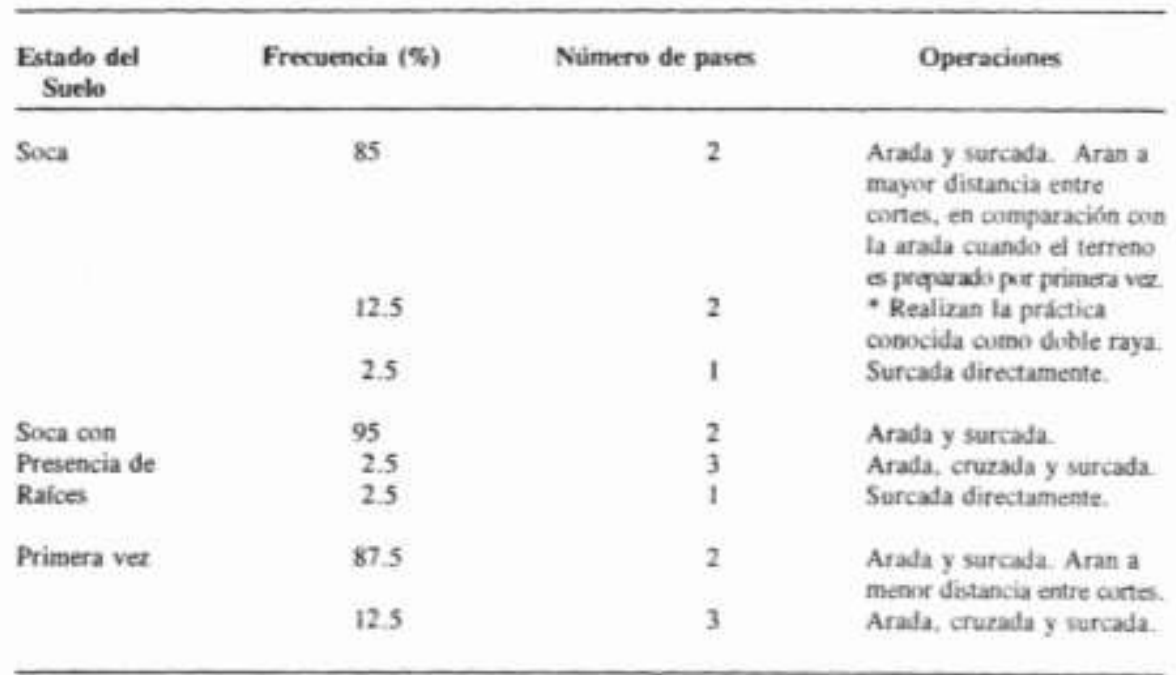

Fuente: Ordóñez M, Serrano M. \& Herrera O (2019) 
Revisemos la fisio cinética del toro para comprender como deber ser el funcionamiento físico del movimiento para poder obtener un cálculo de alta calidad con un porcentaje de error muy bajo. Por ello es necesario trabajar con toros de buena circunferencia escrotal, alta capacidad de servicio y óptima calidad seminal Heritier, M. J. M. (2016).

Figura 5: Diagrama de equilibrio del tren posterior y equilibrio del tren anterior
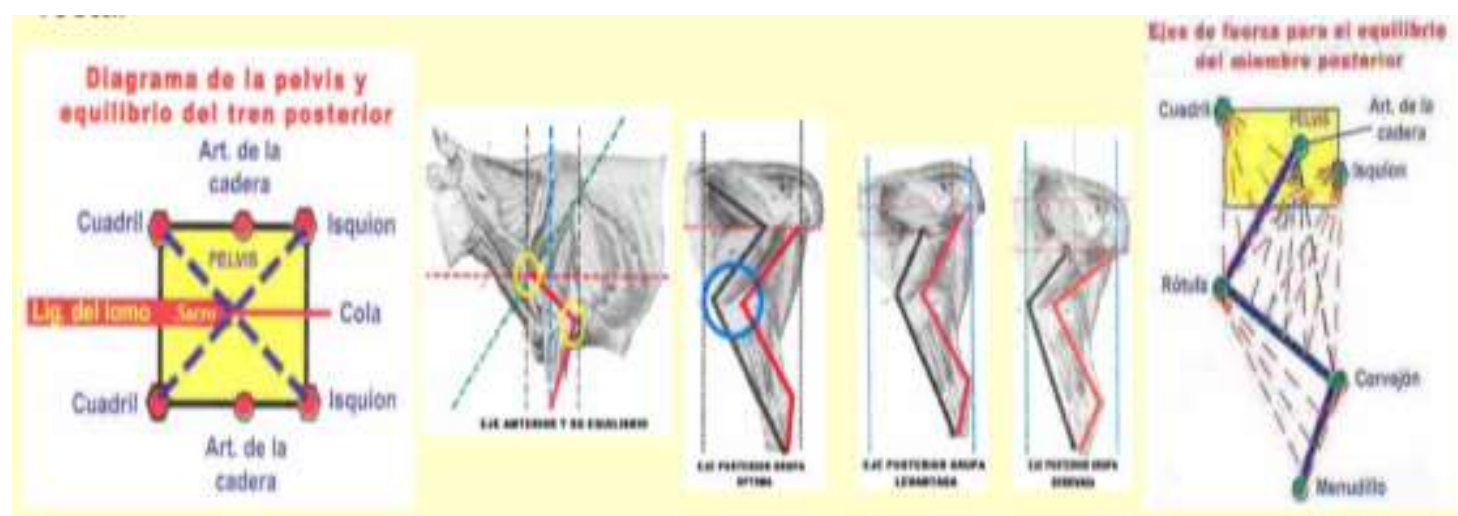

Fuente: Asociación Simmental Simbrah, 2019, mexicana

Cuadro 2: Propiedades de los músculos de toro de arado

PROPIEDADES MECANICAS
ELASTICIDAD: Si al músculo reposo, se le aplica en su extremo una fuerza externa, se distiende, aumenta su longitud, después de que cesa la carga externa recupera su longitud inicial

VISCOSIDAD: Resistencia al movimiento producto de la temperatura de los fluidos que lo componen, Necesidad de calentamiento previo para evitar lesiones RELAJACION: Es la disminución de la fuerza de deformación elástica en el transcurso del tiempo después de una pausa no se aprovechan las fuerzas previas

\section{PROPIEDADES BIOLÓGICAS}


Cuadro 3: Tipos de trabajo muscular

\begin{tabular}{ll}
\hline MOTOR: & $\begin{array}{l}\text { Es cuando el músculo se contrae, tracciones de apoyo, contribuye en el } \\
\text { sentido de movimiento, la longitud de músculo disminuye: Trabajo activo }\end{array}$ \\
\hline RESISTENTE: & $\begin{array}{l}\text { Cuando el músculo, se opone al sentido del movimiento, la longitud del } \\
\text { músculo se alarga, se distiende. Trabajo activo }\end{array}$ \\
ESTÁTICO & $\begin{array}{l}\text { El músculo contribuye a la fijación de algún punto del cuerpo vinculado al } \\
\text { movimiento, tracciones de apoyo su longitud es constante. Trabajo estático }\end{array}$ \\
\hline
\end{tabular}

Fuente: Autores

\section{Análisis matemático para obtener alto rendimiento para el diseño del toro mecánico de alta calidad}

Se considera un peso del toro de $450 \mathrm{Kg}$

Como se indicó anteriormente sabemos que el peso del toro se distribuye el $60 \%$ en la parte frontal y el $40 \%$ en la parte anterior, por lo cual:

PESO PARTE FRONTAL

$$
\begin{gathered}
P_{F}=0.6(450 \mathrm{Kg}) \\
P_{F}=270 \mathrm{Kg}
\end{gathered}
$$

Por lo que el peso en cada pata frontal seria de $135 \mathrm{Kg}$

PESO PARTE ANTERIOR

$$
\begin{gathered}
P_{A}=0.4(450 \mathrm{Kg}) \\
P_{A}=180 \mathrm{Kg}
\end{gathered}
$$

Por lo que el peso en cada pata anterior seria de $90 \mathrm{Kg}$

$$
\begin{gathered}
\sin 40^{\circ}=\frac{\text { CATETO OPUESTO }}{\text { HIPOTENUSA }} \\
\sin 40^{\circ}=\frac{90 \mathrm{Kg}}{\mathrm{H}} \\
H=140 \mathrm{Kgf}
\end{gathered}
$$

$140 \mathrm{Kgf}$ es el peso mínimo que soportara la rodilla del toro de arado. 


\section{Estudio analítico del arado}

No se ha encontrado un estudio serio y completo referido a este tipo de maquinaria agrícola en función de toros o cebús mecánicos que ayuden al hombre en su trabajo en el campo por ello este estudio preliminar para un diseño de este tipo. Rubio Valle, A. E. (2019).

En una tierra compuesta de 70\% de caliza, $20 \%$ de sílice y $10 \%$ de arcilla, con el peso del arado de $40 \mathrm{~kg}$ a $25 \mathrm{~cm}$ de profundidad marco $160 \mathrm{~kg}$, a $17 \mathrm{~cm}$ de profundidad marco $154 \mathrm{~kg}$ y a $13 \mathrm{~cm}$ de profundidad marco $150 \mathrm{~kg}$.

$$
\begin{aligned}
& F_{R \max }=160 \mathrm{KG} \\
& F_{R \min }=150 \mathrm{KG}
\end{aligned}
$$

FUERZA QUE DEBE SOPORTAR LA PATA FRONTAL DEL TORO DE ARADO

$$
\begin{gathered}
F_{F \min }=\left(P_{F}\right)+\left(F_{R \min }\right) \\
F_{F \min }=(135+150) \mathrm{kg} \\
F_{F \min }=285 \mathrm{~kg} \\
F_{F \max }=\left(P_{F}\right)+\left(F_{R \max }\right) \\
F_{F \max }=(135+160) \mathrm{kg} \\
F_{\text {Fmax }}=295 \mathrm{~kg}
\end{gathered}
$$

FUERZA QUE DEBE SOPORTAR LA PATA ANTERIOR DEL TORO DE ARADO

$$
\begin{gathered}
F_{A \min }=\left(P_{A}\right)+\left(F_{R \min }\right) \\
F_{\text {Amin }}=(90+150) \mathrm{kg} \\
F_{A \min }=240 \mathrm{~kg} \\
F_{\text {Amax }}=\left(P_{A}\right)+\left(F_{R \max }\right) \\
F_{\text {Amax }}=(90+160) \mathrm{kg} \\
F_{\text {Amax }}=250 \mathrm{~kg}
\end{gathered}
$$




\section{$\underline{\text { CALCULO DE VELOCIDADES }}$}

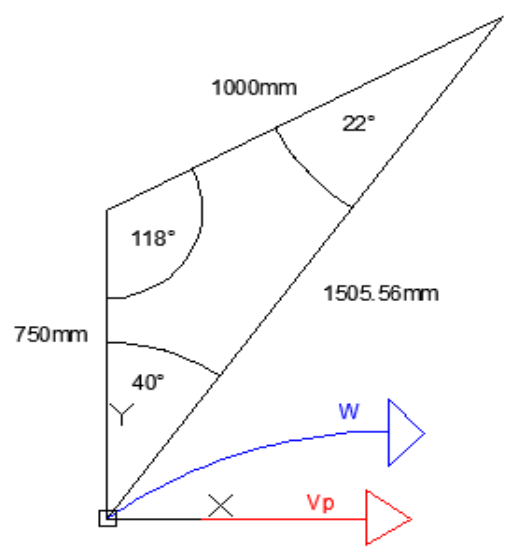

ALTA VELOCIDAD

$$
\begin{gathered}
V_{P}=W * r \\
W=\frac{V_{P}}{r} \\
W=\frac{1 \mathrm{~m} / \mathrm{seg}}{1.6 \mathrm{~m}} \\
W=0.625 \mathrm{rad} / \mathrm{seg}
\end{gathered}
$$

\section{BAJA VELOCIDAD}

1. $V_{P}=W * r$

$$
\begin{gathered}
W=\frac{V_{P}}{r} \\
W=\frac{0.7 \mathrm{~m} / \mathrm{seg}}{1.6 \mathrm{~m}}
\end{gathered}
$$




$$
W=0.4375 \mathrm{rad} / \mathrm{seg}
$$

\section{CALCULO DE ACELERACIONES}

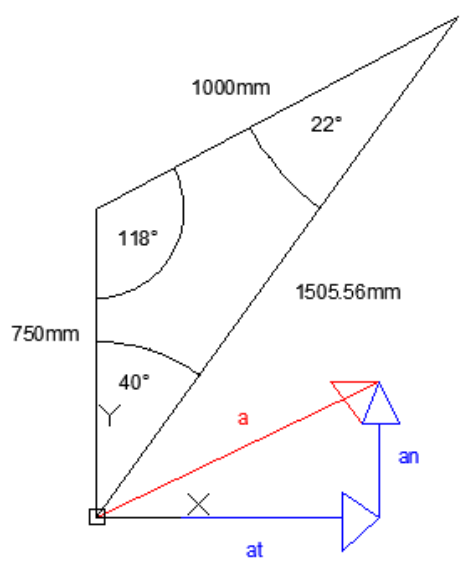

\section{ACELERACION ALTA}

$$
\begin{gathered}
W=W_{0}+\alpha_{c} T \\
\alpha_{c}=\frac{W}{T} \\
\alpha_{c}=\frac{0.625 \mathrm{rad} / \mathrm{seg}}{2 \mathrm{seg}} \\
\alpha_{c}=0.3125 \mathrm{rad} / \mathrm{seg}^{2} \\
a_{\text {tag }}=\left(0.3125 \mathrm{rad} / \mathrm{seg}^{2}\right) *(1.6 \mathrm{~m}) \\
a_{\text {tag }}=\alpha_{c} * r \\
a_{\text {tag }}=0.5 \mathrm{~m} / \mathrm{seg}^{2} \\
a_{n}=\mathrm{w}^{2} * r \\
a_{n}=\left(0.625 \mathrm{rad} / \mathrm{seg}^{2} *(1.6 \mathrm{~m})\right.
\end{gathered}
$$




$$
\begin{gathered}
a_{n}=0.625 \mathrm{~m} / \mathrm{seg}^{2} \\
a=\sqrt{\left(a_{\text {tan }}\right)^{2}+\left(a_{n}\right)^{2}} \\
a=\sqrt{(0.5)^{2}+(0.625)^{2}} \\
a=0.80 \mathrm{~m} / \mathrm{seg}^{2}
\end{gathered}
$$

\section{ACELERACION BAJA}

$$
\begin{gathered}
W=W_{0}+\alpha_{c} T \\
\alpha_{c}=\frac{W}{T} \\
\alpha_{c}=\frac{0.4375 \mathrm{rad} / \mathrm{seg}}{2 \mathrm{seg}} \\
\alpha_{c}=0.2188 \mathrm{rad} / \mathrm{seg}^{2} \\
a_{\text {tag }}=\left(0.2188 \mathrm{rad} / \mathrm{seg}^{2}\right) *(1.6 \mathrm{~m}) \\
a_{\text {tag }}=\alpha_{c} * r \\
a_{\text {tag }}=0.35 \mathrm{~m} / \mathrm{seg}^{2} \\
a_{n}=\left(0.4375 \mathrm{rad} / \mathrm{seg}^{2} *(1.6 \mathrm{~m})\right. \\
a_{n}=0.30 \mathrm{~m} / \mathrm{seg}^{2} \\
\mathrm{w}^{2} * \mathrm{r} \\
(0.35)^{2}+(0.30)^{2}
\end{gathered}
$$




$$
a=0.45 \mathrm{~m} / \text { seg }^{2}
$$

Fuente: Dinámica de Hibbeler décimo segunda edición

\section{Selección de material para el diseño}

Debido a que el material va a tener que soportar fuerzas un tanto elevadas y va a estar sometido a una variación entre la fuerza máxima y la fuerza mínima el material seleccionado para la construcción de nuestro toro de arado biomecánico es un acero ASTM A-514 . Este acero tiene un uso recomendado para estructuras de alta exigencia mecánica y soldabilidad.

A este acero se le puede dar un recubrimiento de cromo para que nos ayude a evitar la oxidación rápida del mismo, debido a que este mecanismo va a estar aplicado en lugares donde el clima es variable, lo cual aumenta la velocidad de oxidación de los materiales.

\section{Selección de mecanismo dinámico}

El mecanismo seleccionado es el MECANISMO DE THEO JANSEN. Que es aplicado en los sistemas de locomoción son claves en ciertas aplicaciones de la robótica como la exploración. Bustamante Téllez, J. (2016).

Este mecanismo está formado por 7 sólidos, de los cuales 5 articulaciones y 2 áreas fijas, como se muestra en la siguiente figura.

Figura 6: Mecanismo de THEO JANSEN

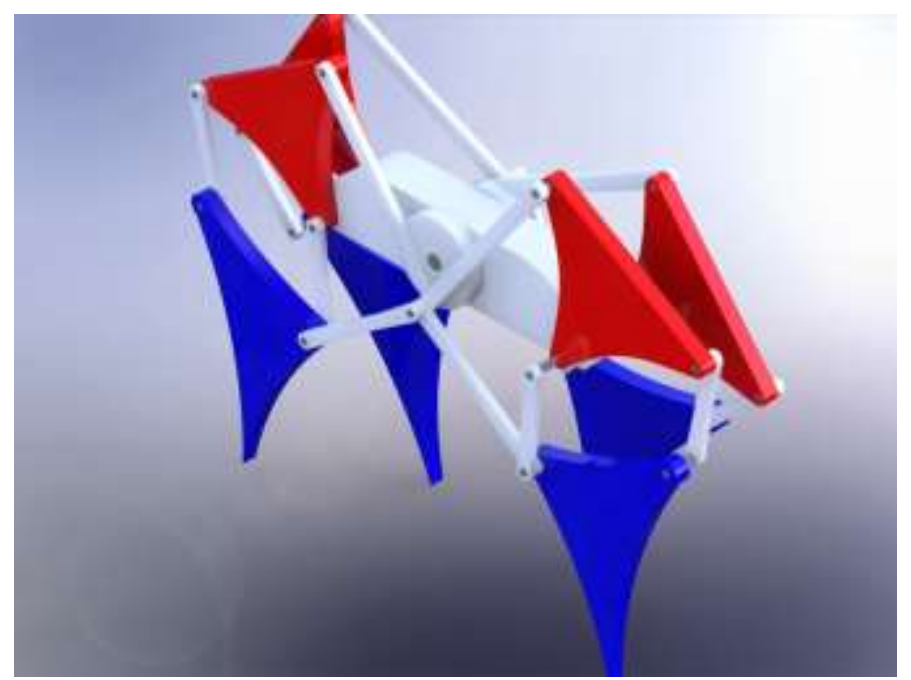

Fuente: Grabcad. (2019) 
El presente trabajo se muestra el desarrollo de una simulación dinámica-cinemática de un mecanismo del tipo Theo Jansen, en donde se desarrolla un estudio de movimiento en contacto con la superficie donde se realiza el desplazamiento del mecanismo. Montejo, A. B., Chávez, A. T., \& Chimal, F. T. (2017).

En la figura 6 debe notarse que el eje al cual está adherido el motor se encuentra en el punto $\mathrm{O}$, además el punto $\mathrm{C}$ es fijo y va con el punto $\mathrm{O}$ alineados en lo que puede llamarse el chasis o cuerpo del animal o mecanismo. El punto A va adherido en la circunferencia o también puede ir en alguna región del círculo lo que genera el movimiento de las extremidades hasta que el punto inferior $\mathrm{G}$ de la pata describa un movimiento como el que se muestra en la figura de la derecha. La ventaja de esta trayectoria es que si el suelo es irregular la distancia entre el mecanismo y el suelo es corta y puede se puede suspender de mejor forma. Este mecanismo hace que sus medidas no puedan ser cualesquiera, sino que han de ser prácticamente a escala del original; aunque permite pequeñas modificaciones para optimizar la trayectoria del punto $\mathrm{G}$ en función de los pasos de la máquina.

\section{Conclusiones.}

- Se realizó los cálculos correspondientes de cada una de las fuerzas que actúan en las extremidades inferiores del animal, las extremidades posteriores y anteriores obteniendo unos valores máximos de $295 \mathrm{~kg}$ en la extremidad frontal y $250 \mathrm{~kg}$ en la extremidad anterior.

- De forma matemática y precisa se determinó las aceleraciones y velocidades optimas par aun buen desempeño del prototipo de toro de arado biomecánico estableciendo un rango para cada una de estas propiedades, determinando que la aceleración ha de estar en un rango de $0.45 \frac{\mathrm{m}}{\mathrm{s}^{2}}-0.8 \frac{\mathrm{m}}{\mathrm{s}^{2}}$ y un rango de velocidad de $0.4375 \frac{\mathrm{rad}}{\mathrm{s}}-0.625 \frac{\mathrm{rad}}{\mathrm{s}}$

- Se comprobó el mejor mecanismo de accionamiento para obtener la mejor ventaja mecánica y estabilidad del toro asegurando así su óptimo rendimiento y posibles volcamientos, siendo el mecanismo de Theo Jansen el cual ubica de forma estratégica y adecuada la fuente de movimiento de la maqueta.

- Establecimos el material más adecuado para la construcción del toro de arado tomando en cuenta las fuerzas, velocidades, aceleración y mecanismo de accionamiento siendo el acero ASTM A-514.

\section{Referencias bibliográficas.}

Alberdi, M. T., \& Prado, J. L. (1995). Los équidos de América del Sur. Evolución climática y biológica de la región pampeana durante los últimos cinco millones de años. Un ensayo de correlación con el Mediterráneo occidental (Alberdi, MT, 295-308. 
Arciniegas, J. G., \& Lacki, P. (1993). La modernizacion de la agricultura: los pequenas tambien pueden. FAO-Oficina Regional para America Latina y el Caribe.

Barreto Andrade, J., Villarroya-Aparicio, A., \& Calero Morales, S. (2017). Biomecánica de la marcha atlética. Análisis cinemático de su desarrollo y comparación con la marcha normal. Revista Cubana de Investigaciones Biomédicas, 36(2), 53-69.

Barreiro, M. W. (2017). Evaluación de tecnologías de preparación de suelos con tracción animal. Revista Ingeniería Agrícola, 6(1), 9-13.

Bustamante Téllez, J. (2016). Diseño de mecanismo de locomoción andante con cambio de dirección.

Chirgwin, J. C. (1995). Los animales de trabajo y el desarrollo sostenible. World Anim Review (FAO), 1014-6954.

Elvira, J. L. L., Garcia, F. J. V., Meana, M., \& García, J. A. (2008). Análisis biomecánico del apoyo plantar en la marcha atlética: relación entre la huella plantar, ángulos de la articulación subastragalina y presiones plantares. European Journal of Human Movement, (20), 41-60.

Fernández-Baca,S. (1991). Avances y perspectivas del conocimiento de los camélidos sudamericanos (No. QL 737. U54. A92 1991).

González Valdés, R., García de la Figal Costales, A. E., Morejón Mesa, Y., \& Morales

Heritier, M. J. M. (2016). Características de Toros Aptos en la Región Semiárida Central.

Hetz, E., \& Pérez, R. (1994). Rendimiento físico, requerimientos energéticos y adaptación fisiológica del caballo de tiro en faenas de aradura. Archivos de Medicina Veterinaria, 26(2), 15.

Izquierdo, M., \& Redín, M. I. (2008). Biomecánica y bases neuromusculares de la actividad física y el deporte. Ed. Médica Panamericana.

Machuca Mendoza, C. R., \& López Ayala, L. F. (2015). Locomoción de un robot cuadrúpedo basada en redes neuronales artificiales (Bachelor's thesis, Escuela Superior Politécnica de Chimborazo).

Mehuys, G. R., Tiessen, K. H., Villatoro, M., Sancho, F., \& Lobb, D. A. (2009). Erosión por labranza con arado de disco en suelos volcánicos de ladera en Costa Rica. Agronomía Costarricense Vol. 33 Núm. 22009.

Montejo, A. B., Chávez, A. T., \& Chimal, F. T. (2017). ANÁLISIS CINEMÁTICO Y DINÁMICO DE UN MECANISMO DE THEO JANSEN. JÓVENES EN LA CIENCIA, 3(1), 484-489.

Ordóñez, M. V., López, M. S., \& Gutiérrez, O. A. H. (1995). Diagnóstico del uso de animales de tiro en una zona montañosa del norte del Cauca. Acta Agronómica, 45(2-4), 97108.

Rodríguez, D. (2009). Evaluación energética de la labor de rotura con tracción animal y tractor MTZ-510. Estudio de caso: Granja Guayabal, San José de las Lajas, La Habana, Cuba. Revista Ciencias Técnicas Agropecuarias, 18(3).

Rubio Valle, A. E. (2019). Diseño y optimización de un arado reversible por gravedad. 
Mejías J. (2014). Anatomia del pie bobino.[Figura] Recuperado de https://image.slidesharecdn.com/anatomiadelpiebovinoft-140407134211phpapp02/95/anatomia-del-pie-bovino-ft-16-638.jpg?cb=1396878164

Gobierno de México, Yunta: ¿de mulas o de bueyes? (2019 recuperado de: https://www.gob.mx/siap/articulos/yunta-de-mulas-o-de-bueyes

García J. (2019) recuperado de https://es.slideshare.net/juanmejias545/anatomia-del-piebovino-ft?qid=dc5d087e-2c5e-4591-b475dc6720068571\&v=qf1\&b\&from_search=1

García J (2019) recuperado de https://es.slideshare.net/juanmejias545/anatomia-del-piebovino-ft?qid=dc5d087e-2c5e-4591-b475-dc6720068571\&v=qf $1 \& b \&$ from_search=1

Ivot (2019) recuperado de http://www.traumatologiaveterinaria.com/articulaciones/docs/biomecanica.pdf

Rodriguez E (1986) Recuperado de https://books.google.com.ec/books?id=TtDdk88cDnMC\&pg=PA33\&lpg=PA33\&dq $=$ peso+de+un+buey+de+tiro\&source $=$ bl\&ots $=$ GjY6vH8Uhw $\&$ sig $=$ PFEkyVYIl WTbicjh8XN2W04r3g\&hl=es-419\&sa=X\&ved=0ahUKEwi27ii6eHJAhUJWCYKHXhfAf8Q6AEILjAE\#v=onepage\&q=peso $\% 20 \mathrm{de} \% 20 \mathrm{un} \% 2$ 0buey\%20de\%20tiro\&f=false

Ordóñez M, Serrano M. \& Herrera O (2019) recuperado de http://www.bdigital.unal.edu.co/19589/1/15597-47584-1-PB.pdf

Asociación simmental simbrah, 2019, mexicana http://www.simmentalsimbrah.com.mx/pdf/Seleccion\%20de\%20un\%20Semental.p df

Grabcad. (2019) https://grabcad.com/library/mecanismo-theo-jansen-2

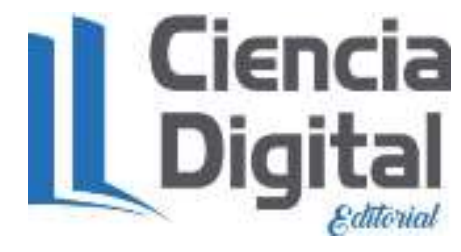




\section{PARA CITAR EL ARTÍCULO INDEXADO.}

Cruz Siguenza, E., Villagrán Cáceres, W., Arguello, E., \& Abarca Pérez, E. (2019). Análisis matemático para el diseño de Toro de Arado Biomecánico de alta Calidad. Ciencia Digital, 3(2), 689-701. https://doi.org/10.33262/cienciadigital.v3i2.478

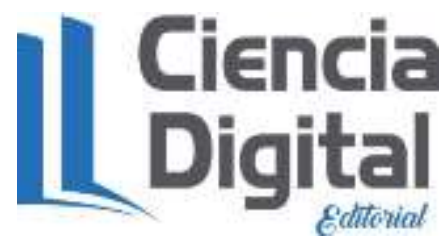

El artículo que se publica es de exclusiva responsabilidad de los autores y no necesariamente reflejan el pensamiento de la Revista Ciencia Digital.

El artículo queda en propiedad de la revista y, por tanto, su publicación parcial y/o total en otro medio tiene que ser autorizado por el director de la Revista Ciencia Digital.
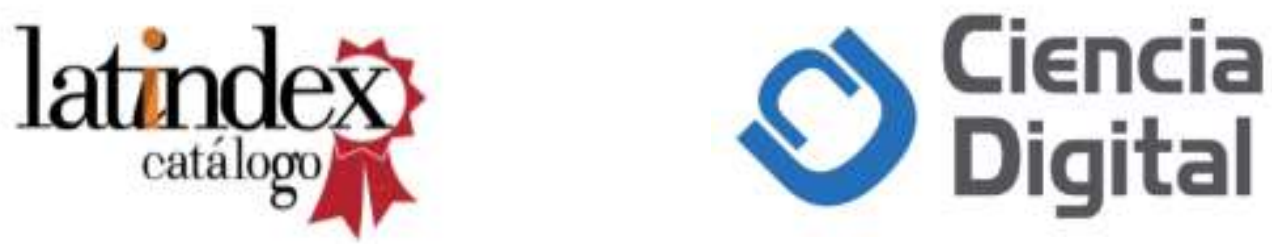